\title{
VALIDITY OF DIFFUSION WEIGHTED MAGNETIC RESONANCE IMAGING AND APPARENT DIFFUSION COEFFICIENT MAP IN DIFFERENTIATING BENIGN FROM MALIGNANT UTERINE ENDOMETRIAL PATHOLOGIES
}

\author{
Marwa Majid Al-Adhab ${ }^{*}$, Salam Mohammad Joori ${ }^{\circledR}$ \& Enam Azez Al- \\ Tameemi ${ }^{\#}$ \\ *MB,ChB, FIBMS (Diagnostic Radiology), lecturer at Department of Surgery, College of Medicine, \\ University of Basrah. ${ }^{\circledR} \mathrm{MB}, \mathrm{ChB}, \mathrm{DMRD}, \mathrm{FJMC}(\mathrm{RAD})$, Consultant Radiologist, Radiology Institute, \\ Medical City, Baghdad. "MB,ChB, DMRD, Radiology Specialist, Oncology Teaching Hospital, Medical \\ City, Baghdad, IRAQ.
}

\begin{abstract}
Endometrial pathologies represent a diagnostic challenge for radiologist and gynecologist due to dynamic changes of the endometrium, wide variability in imaging appearance and overlap between benign and malignant causes of endometrial abnormalities. Although tissue analysis via dilatation and curettage, endometrial biopsy or hysteroscopy is the backbone in the diagnosis, these tests are invasive, not without complications and may be difficult to perform in certain circumstances, hence the need for noninvasive imaging methods to aid in the diagnosis and triaging the patient for subsequent invasive procedures as well as contribution in treatment planning.

This study aimed to evaluate the role of diffusion weighted imaging (DWI) with apparent diffusion coefficient (ADC) value measurement in differentiating benign from malignant uterine endometrial lesions.

The study included 47 patients with endometrial lesions divided into two groups according to the result of histopathological analysis; the malignant group consisting from 18 cases and the benign group consisting from 29 cases, the latter was further subdivided into: polyp, hyperplasia and other benign entities. Pelvic magnetic resonance imaging with DWI performed for each patient with visual evaluation of signal intensity on diffusion and ADC value measurement. Subsequently mean ADC values for each group were calculated and compared, and validity measures for the optimal cut-off values for differentiating benign from malignant lesions were determined.

The mean \pm standard deviation for ADC value $(x 10-3 \mathrm{~mm} 2 / \mathrm{sec})$ for malignant group was $0.71 \pm 0.12$, and for benign group was $1.52 \pm 0.42$, with a significant difference between the two groups ( $P$ value $<0.01$ ), there was no significant difference in ADC value between the subdivision of benign group. At ADC cut-off value of $0.976 \times 10-3 \mathrm{~mm} 2 / \mathrm{sec}$, the sensitivity, specificity, positive predictive value and negative predictive value and accuracy of DWI in detecting endometrial carcinoma were $100 \%, 89.6 \%, 85.71 \%, 100 \%$ and $93.62 \%$ respectively.

In conclusion, DWI with ADC value measurement is a valuable non-invasive diagnostic test, aiding in the differentiation of benign from malignant uterine endometrial cavity lesions.

Key words: diffusion weighted imaging, apparent diffusion coefficient map, benign endometrial lesions, malignant endometrial lesions.
\end{abstract}

\section{Introduction}

G ndometrial pathologies represent a diagnostic challenge for radiologist and gynecologist due to dynamic changes of the endometrium affected by patient age, state of menstrual cycle and use of hormonal replacement therapy in addition to wide variability in imaging appearance and overlap between benign and malignant causes of endometrial abnormalities. Although tissue diagnosis via dilatation and curettage (D\&C), endometrial biopsy or hysteroscopy is the 
backbone in the diagnosis these tests remain an invasive procedures, not without complications and may be difficult to perform in certain circumstances like vaginal or cervical stenosis, morbid obesity, a lot of comorbidities, clotting disorder, acute cervicitis or in women without sexual experience, in addition, these tests may be inconclusive due to sampling errors, hence the need for noninvasive imaging methods to aid in the diagnosis and triaging the patient for subsequent invasive procedures in addition to contribution in treatment planning and follow up ${ }^{1-3}$.

Magnetic resonance imaging (MRI) role in gynecologic oncology has evolved during the past years, MRI has been shown to be superior to computed tomography (CT) in staging of gynecological malignancies, likewise, there is evidence that MRI may aid in differentiating recurrent tumor from radiation fibrosis, the advantages of MRI include its excellent spatial and tissue contrast resolution, multiplanar capability, lack of ionizing radiation, its capability to perform dynamic imaging allowing for functional evaluation, in addition, MRI has been shown to minimize cost in some clinical settings by limiting or eliminating the need for further expensive or more invasive diagnostic or surgical procedures ${ }^{4,5}$.

Functional imaging is becoming increasingly important in the assessment of patients with cancer because of the limitations of morphologic imaging, diffusion weighted imaging (DWI) which is a special sequence of MRI- is one of these functional imaging, it had been established as a useful tool in neurologic applications for many years, but recent technical advances allow its use in abdominal and pelvic applications ${ }^{6}$. The principle of DWI involve utilization of water movement in the intra- and extracellular spaces and vessels. In a totally unrestricted environment, water movement would be completely random, a phenomenon known as Brownian motion, however, within biologic tissues, the movement of water is not completely random, instead its impeded by interaction with tissue compartments, intracellular organelles and cell membranes, the presence of intact cell membranes and the extent of tissue cellularity help determine the impedance of water molecules diffusion. Certain tissue types have been reported to be associated with impeded diffusion including tumor, abscess, cytotoxic edema and fibrosis. Tissues with low cellularity or those consisting of cells with disrupted membranes allow greater movement of water molecules ${ }^{7,8}$.

The DWI is based on the standard spin echo T2 weighted sequence which in turn consists of $90^{\circ}$ and $180^{\circ}$ radio frequency (RF) pulse with the T2 decay depending on transverse relaxation. The presence of water diffusion is observed as signal loss on $\mathrm{DWI}^{7,9,10}$.

The strength of diffusion sensitizing gradient is expressed as b value, adjustment of $b$ value results in adjustment of sensitivity of diffusion sequence ${ }^{7,11,12}$.

At high $b$ value, a region of high signal intensity (SI) suggests restricted diffusion this usually occurs in highly cellular tissues due to tight packing of water molecules in these tissues. In clinical practice, the signal loss in water molecules at different $b$ values can be utilized for detection and characterization of different lesions ${ }^{7,11}$.

Evaluation of DWI Quantitatively is done by using apparent diffusion coefficient (ADC) map that is calculated during post processing with the use of at least two $b$ values, this analysis is an automated process available as an application on workstation. ADC map is displaced parametrically as gray scale images, by drawing regions of interest within a region the ADC value for this region can be determined, its measured in 
$\mathrm{mm} 2 / \mathrm{sec}^{6,7,13}$. Since the SI detected on DWI depends on both $\mathrm{T} 2$ relaxation time and water diffusion, an area with very long $\mathrm{T} 2$ relaxation time can still have high signal on DWI and erroneously considered as area of restricted diffusion, this effect is known as T2 shine through. As ADC is independent of magnetic field strength it can overcome T2 shine through effect, as such, an area of true restricted diffusion will show low ADC value (darker shade of gray) compared with that of low cellularity (e.g. within cysts) which shows high ADC value (higher shade of gray) $^{6,11}$.

We need to keep in mind that ADC maps have poor anatomical details and its interpretation should be done in conjunction with other sequences including DWI, T2 weighted image, T1 weighted image and contrast-enhanced images if available ${ }^{13}$.

The main advantages of DWI are the ability to improve lesion detection, which in turn increase the sensitivity, and the opportunity for further lesion characterization leading to increased specificity. The technique often results in high lesion to background contrast leading to increase lesion conspicuity and thus improve its detection. These advantages are emphasized when contrast cannot be given for example due to severe renal impairment or allergy, or the obtained post contrast images do not offer a diagnosis (for example due to motion artifact, improper bolus timing, baseline hyperintense lesion on $\mathrm{T} 1$, or in cases of too small lesion difficult to characterize), in these circumstances DWI and ADC value become valuable and even when post contrast images are available the DWI and ADC value can increase the radiologist's confidence or reinforce information provided by other sequences in the protocol ${ }^{14,15}$.

The aim of this study is to evaluate the role of diffusion weighted imaging with apparent diffusion coefficient value measurement in differentiating benign from malignant uterine endometrial pathologies.

\section{Patients and Methods}

This cross-sectional analytic study had been conducted in the MRI unit in Oncology teaching hospital of Baghdad medical city during the period from January 2017 to December 2017. The study is approved by our ethical and scientific committee, and verbal consents had been taken from all patients.

Inclusion criteria: Patients with sonographic evidence of thick endometrium or focal endometrial pathology whether symptomatic or asymptomatic. This include both postmenopausal and premenopausal patients. The uniform endometrium is considered thick if it is $\geq 5 \mathrm{~mm}$ in postmenopausal women, while in premenopausal women a thickness that is not compatible with the expected thickness in that phase of menstrual cycle was considered abnormal (i.e. $>8 \mathrm{~mm}$ in proliferative phase and $>16 \mathrm{~mm}$ in secretory phase). Any focal thickening, mass or heterogeneity also considered abnormal regardless of the threshold.

Exclusion criteria: General contraindication for MRI (like pacemaker or metallic implant), Claustrophobic patients or those unable to cooperate, Patients with no histopathological proof, Patients with non-conclusive MRI examination.

During the period of the study, 67 patients were referred to our MRI unit from gynecologic or oncologic outpatient clinics with a clinical suspicion of endometrial pathology and had undergone pelvic MRI examination with diffusionweighted imaging. After applying the exclusion criteria our convenient sample consisted from 47 patients with a mean age of 57.13 years (minimum 42 years, maximum 74 years).

The tissue sample for histopathological analysis was obtained either by surgical resection in form of total abdominal 
hysterectomy and bilateral salpingooophorectomy (25 patients), by D\&C (21 patients), or by hysteroscopic-guided resection (one patient).

MRI Image Acquisition.

The MR examinations were performed with a 1.5 Tesla MR system (Magnetom Aera; Siemens Healthineers, Erlangen, Germany) using body 18 matrix coil (18channel design with 18 integrated preamplifiers, with 3 rows of 6 elements each) placed on the pelvic region.

For each patient referred to us, simple information regarding age, clinical presentation, marital state, parity, comorbidity had been collected. We tried to ensure that there was no active bleeding at time of examination (otherwise the examination postponed). Then the following pelvic MRI protocol had been done in supine position: Axial fast spinecho T2 WI, sagittal fast spine-echo T2 WI, Axial T2 WI with fat suppression, Axial T1 WI, Sagittal T1 WI, DWI at b value 50, 400 and $800 \mathrm{sec} / \mathrm{mm} 2$ using two-dimensional echo planer imaging (EPI) under free breathing, the acquisition of DWI was in both sagittal and axial plane in seven patients and only in sagittal plane in 40 patients. From our observations, the sagittal plane was the best to demonstrate most of the endometrial cavity on the same section allowing better interpretation of the SI and easier correlation with other sequences, and since there is no difference in ADC value measured by either plane hence we depend mostly on DWI in sagittal plane and omit the axial plane to save time. The ADC map will be automatically reconstructed from the above $b$ values on the syngo.via workstation (software version: syngo MR $E$ 11). Then we performed contrast enhanced axial and sagittal T1 WI using Dimeglumine gadopentetate $469 \mathrm{mg}$ (Magnevist ${ }^{\circledR}$ ) as contrast agent administered intravenously (IV) by manual injection at a dose of $0.1 \mathrm{mmol} / \mathrm{kg}$ body weight, the imaging parameter was the same as for unenhanced T1WI, this sequence had been done in all patient apart from four (two of them had impaired renal function, the result of renal function was not available in one patient, and one refused the IV contrast). Total pelvic MR examination time was about 15 minutes and $13 \mathrm{sec}$, the imaging parameters for each sequence are illustrated in table I.

Table (I): imaging parameters for each MRI sequence

\begin{tabular}{|c|c|c|c|c|c|c|c|c|c|c|}
\hline Sequence & $\begin{array}{l}\text { FOV* } \\
(\mathrm{mm})\end{array}$ & $\begin{array}{l}\text { Acquis. } \\
\text { Matrix }\end{array}$ & Slices & $\begin{array}{l}\text { Slice } \\
\text { thickness }\end{array}$ & $\begin{array}{l}\mathrm{TR}^{\wedge} \\
(\mathrm{ms})\end{array}$ & $\begin{array}{l}\text { TE } \infty \\
(\mathrm{ms})\end{array}$ & Average & $\begin{array}{l}\begin{array}{l}\text { Phase } \\
\text { over- } \\
\text { sampling }\end{array} \\
\end{array}$ & $\begin{array}{l}\text { Voxel } \\
\text { size } \\
(\mathrm{mm})\end{array}$ & $\begin{array}{l}\text { Acquis. } \\
\text { time } \\
(\mathrm{sec}) \\
\end{array}$ \\
\hline $\begin{array}{l}\text { Axial fast spine } \\
\text { echo T2 WI }\end{array}$ & 360 & $208 \times 320$ & 33 & $5 \mathrm{~mm}$ & 1400 & 89 & 1 & $0 \%$ & $\begin{array}{l}1.2 \mathrm{x} \\
1.2 \times 5 \\
\end{array}$ & 52 \\
\hline $\begin{array}{l}\text { Sagittal fast spine } \\
\text { echo T2 WI }\end{array}$ & 300 & $240 \times 320$ & 22 & $5 \mathrm{~mm}$ & 1400 & 93 & 1 & $30 \%$ & $\begin{array}{l}0.9 \mathrm{x} \\
0.9 \mathrm{x} 5 \\
\end{array}$ & 34 \\
\hline $\begin{array}{l}\text { Axial T2 with fat } \\
\text { suppression }\end{array}$ & 380 & $208 \times 256$ & 30 & $6 \mathrm{~mm}$ & 1400 & 91 & 1 & $0 \%$ & $\begin{array}{l}1.5 \mathrm{x} \\
1.5 \mathrm{x} 6 \\
\end{array}$ & 44 \\
\hline Axial T1WI & 220 & $240 \times 320$ & 28 & $4 \mathrm{~mm}$ & 550 & 20 & - & - & $\begin{array}{l}0.7 \mathrm{x} \\
0.7 \mathrm{x} 4\end{array}$ & 179 \\
\hline Sagittal T1 & 250 & $256 \times 320$ & 26 & $4 \mathrm{~mm}$ & 584 & 21 & 2 & $50 \%$ & $\begin{array}{l}0.8 \mathrm{x} \\
0.8 \mathrm{x} 4 \\
\end{array}$ & 152 \\
\hline DWI & 38 & $115 \times 192$ & 30 & $6 \mathrm{~m}$ & 4800 & 68 & & $10 \%$ & $1 \times 1 \times 6$ & 186 \\
\hline
\end{tabular}

* FOV: field of view, ${ }^{\wedge}$ TR: time to repeat, $\infty$ TR: time to echo

Image Interpretation: To start with, a general evaluation of the morphological features and signal intensity of the endometrium was done on all pulse sequences including assessment of its thickness, homogeneity of the signal intensity, presence of any focal lesion within endometrial cavity, the sharpness of the interphase with myometrium and any myometrial invasion. Then qualitative analysis of endometrial signal intensity on DWI sequence was done 
visually depending on the highest $b$ value $(800 \mathrm{sec} / \mathrm{mm} 2)$ and the lesion had been classified as hyperintense if its SI was higher than the myometrium and hypo or iso intense if its SI was lower or equal to that of myometrium respectively, during statistical analysis both hypointense and isointense lesions gathered into one group as both indicate absence of restricted diffusion.

Quantitative analysis was done on ADC map by drawing the circular region of interest (ROI) in a representative area within the lesion and to maximize the accuracy of measurement, reference to findings in other imaging sequences (specially DWI) had been done to choose an area within the lesion that shows the highest SI on DWI, the darkest on ADC map, and trying to avoid areas of inhomogeneity, artifacts, T2 blackout, hemorrhagic foci and area of cystic changes. We also tried to avoid the pedicle of the polyp if visible. The measurement had been repeated many times (three on average) and the mean value had been calculated from these measurements and expressed as (..x 10-3 $\mathrm{mm} 2 / \mathrm{sec})$. The interpretation had been done by two radiologists blinded to the result of histopathological analysis.

The mean ADC value for each lesion had been recorded then according to the histopathological diagnosis patients were classified into two major groups: benign and malignant, the benign group was further sub divided into those due to polyp, hyperplasia or other benign entities not otherwise specified (dysfunctional hormonal imbalance proliferative endometrium glandular pattern, secretory phase endometrium or non-secretory endometrium).

Data were entered and analyzed with IBM SPSS statistical package. Continuous variables were expressed as mean \pm standard deviation (SD) with their confidence intervals being represented as (lower limit-upper limit), while categorical variables were expressed as frequencies and percentages. Continuous data were explored both for normality of distribution utilizing Shapiro-Wilk and Kolmogorov-Smirnov tests of normality, and for equality of variance both between groups of benign and malignant lesions and among different types of benign lesions with Levene's test of equality of variance. Continuous variables that were normally distributed were further analyzed with parametric tests. Since in our data the ADC values for each of benign and malignant groups were normally distributed, an unpaired student $t$ test was used. Similarly, one-way analysis of variance (ANOVA) was implemented in the comparison of means of ADC values among distinct groups of benign lesions as they were normally distributed in each category as well.

Subsequently, to determine the utility of $\mathrm{ADC}$ value in differentiating benign from malignant endometrial lesions and to calculate its optimal cut-off value(s), receiver operator characteristics (ROC) curve analytics were performed. Beside sensitivity and specificity, other measures of validity, namely false positive and false negative rates, positive and negative predictive values in addition to accuracy were calculated for the cut-off value(s) that were regarded as optimal (i.e., yielded the most optimal sensitivity and specificity combined). All significance tests were two tailed and results were considered statistically significant when the $\mathrm{P}$ value is less than 0.05 .

\section{Results}

This study included 47 patients with mean age of $57.13 \pm 9.68$ years, the youngest being 42 years and the oldest being 74 years, 10 of them were premenopausal $(21.3 \%)$, and 37 patients were in post-menopausal state $(78.7 \%)$, we divided the patients into two groups according to the result of histopathological analysis:

Benign group: composed from 29 patients (61.7\% of total cases) mean age $59 \pm 9.55$ 
years (minimum 42 years, maximum 74 years), from them 21 patients $(72.4 \%)$ presented with vaginal bleeding (AUB), seven patients $(24.1 \%)$ with abdominal pain and one patient $(3.4 \%)$ discovered incidentally to have thick endometrium, this group is further subdivided into three subgroups according to the type of benign lesion: polyp in 13 patients (45\% of the total benign lesions) six of them diagnosed by $\mathrm{D} \& \mathrm{C}$, one by hysteroscopic resection and the rest by hysterectomy, hyperplasia in 10 patients $(34 \%)$ six of them diagnosed by D\&C and the rest by hysterectomy and other benign entities in six patients $(21 \%)$ dysfunctional hormonal imbalance proliferative endometrium glandular histological pattern in three patients, secretory phase changes in one patients, non-secretory endometrial glands in two patients, two of these patients were diagnosed by $\mathrm{D} \& \mathrm{C}$ while the rest by hysterectomy.

Malignant group: This group is composed from 18 patients $(38.3 \%$ of total cases) mean age $54 \pm 9.32$ years (minimum 42 years, maximum 72 years), all of them $(100 \%)$ present with vaginal bleeding, on histopathological analysis $88.8 \%$ of lesions (16 lesions) were of well differentiated endometrioid type endometrial carcinoma and $11.1 \%$ (two lesions) were of type II endometrial carcinoma (high grade papillary serous carcinoma in one case and mixed endometrioid and high grade clear cell subtype in the second case).

The mean endometrial thickness in benign group was $15.19 \pm 8.34 \mathrm{~mm}$ (range from 4 to $40 \mathrm{~mm}$ ) and in the malignant group $26.83 \pm 12.186 \mathrm{~mm}$ (range from 12 to $55 \mathrm{~mm}$ ).

All lesions in malignant group (18 lesions) show hyperintense signal on DWI while 15 lesions from benign group $(51.7 \%)$ show hyperintense signal and 14 lesions show iso/hypointense signal. Further details on imaging characteristic on T2, DWI and the presence of irregularity in endometrial-myometrial junction or myometrial invasion are illustrated in table II.

Table II: Imaging characteristics of benign and malignant lesions.

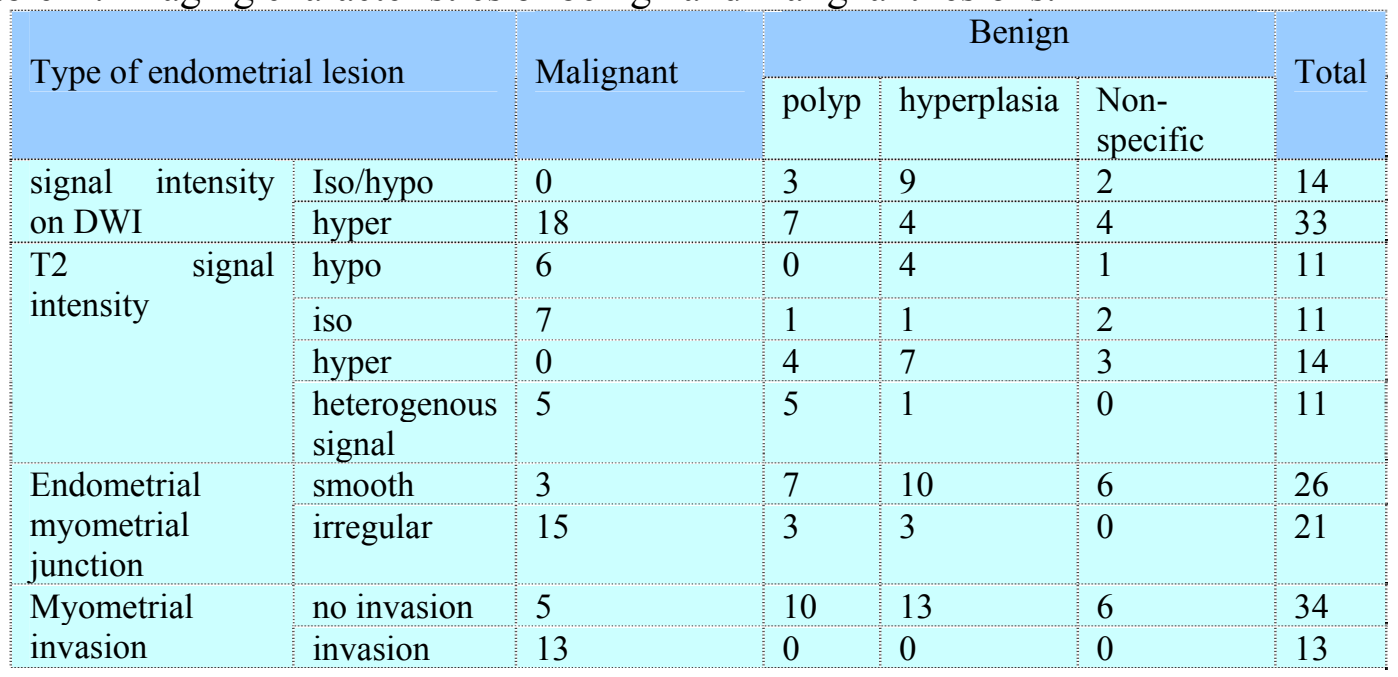

The mean ADC value for benign group was $1.52 \times 10-3 \pm 0.42 \mathrm{~mm} 2 / \mathrm{sec}$, and for malignant group was $0.71 \times 10-3 \pm 0.12 \mathrm{~mm} 2 / \mathrm{sec}$ with a significant difference between the two groups $(\mathrm{P}<0.01)$ as shown in table III. 
Table III: T-test statistics for mean difference between benign and malignant lesions.

\begin{tabular}{|l|l|l|l|l|l|l|l|}
\hline $\begin{array}{l}\text { Type of } \\
\text { the lesion }\end{array}$ & $\begin{array}{l}\text { Mean ADC* } \\
\text { value (x10-3 } \\
\text { mm2/sec) }\end{array}$ & $\begin{array}{l}\text { Standard } \\
\text { Deviation }\end{array}$ & $\begin{array}{l}\text { Standard } \\
\text { Error of } \\
\text { mean }\end{array}$ & $\begin{array}{l}\text { Mean } \\
\text { difference }\end{array}$ & $\begin{array}{l}95 \% \mathrm{CI}^{\wedge} \\
\text { mean } \\
\text { difference }\end{array}$ & $\begin{array}{l}\mathrm{T} \\
\text { Value }\end{array}$ & $\begin{array}{l}\mathrm{P} \\
\text { Value }\end{array}$ \\
\hline Benign & 1.518 & 0.424 & 0.079 & 0.805 & $(0.635-$ & 9.606 & $<0.01$ \\
\hline Malignant & 0.713 & 0.121 & 0.029 & & $0.975)$ & & \\
\hline
\end{tabular}

*ADC: apparent diffusion coefficient,${ }^{\wedge} \mathrm{CI}$ : confidence interval

With regards to subdivisions of the benign group, as revealed on one-way ANOVA test, there was no statistically significant difference in the mean ADC value of hyperplasia, polyp and other benign endometrial lesions $(\mathrm{P}=0.342)$ (table IV).

Table IV: Comparison of ADC value for each subdivision of the benign group.

\begin{tabular}{|l|l|l|l|l|}
\hline $\begin{array}{l}\text { Type of benign } \\
\text { endometrial } \\
\text { lesion }\end{array}$ & $\begin{array}{l}\text { Mean ADC* value } \\
(\mathrm{x} 10-3 \mathrm{~mm} / \mathrm{sec})\end{array}$ & $\begin{array}{l}\text { Standard } \\
\text { deviation }\end{array}$ & $\begin{array}{l}\text { One-way ANOVA } \\
\text { statistics (F value) }\end{array}$ & P Value \\
\hline Hyperplasia & 1.61 & 0.39 & & \multirow{2}{*}{0.342} \\
\hline Polyp & 1.55 & 0.49 & 1.119 & \\
\hline Others & 1.29 & 0.26 & & \\
\hline
\end{tabular}

*ADC: apparent diffusion coefficient

Upon ROC curve analysis of different ADC cut-off values for differentiation between benign and malignant endometrial lesions, the area under the curve was (0.994) with a $P$ value $<0.01$, this indicates that ADC value estimation in DWI is an excellent tool for differentiation between benign and malignant lesions. According to ROC curve the two most suitable cut off ADC values are $0.976 \times 10-3$ and $0.874 \times 10-3 \mathrm{~mm} 2 / \mathrm{sec}$, details on their sensitivity, specificity and positive and negative predictive values are shown in table V.

Table V: Validity of ADC value in differentiating benign from malignant endometrial lesions.

\begin{tabular}{|l|l|l|l|l|l|}
\hline ADC* Cut off value & Sensitivity & Specificity & PPV* & NPV* & Accuracy \\
\hline 0.976 & $100 \%$ & $89.6 \%$ & $85.71 \%$ & $100 \%$ & $93.62 \%$ \\
\hline 0.874 & $94.44 \%$ & $100 \%$ & $100 \%$ & $96.67 \%$ & 97.87 \\
\hline *ADC: Apparent diffusion coefficient. *PPV: Positive predictive value \\
*NPV: Negative predictive value
\end{tabular}

\section{Discussion}

Diffusion weighted imaging is one of imaging biomarkers that depends on microscopic motion of water (Brownian motion), this motion is highly affected by the cellular environment of water thus DWI could be an early indicator of biological abnormalities, in addition the acquisition is rapid, non-invasive, noncontrast reliant and with no use of ionizing radiation yet still quantitative and easily included in the routine imaging protocol $^{16}$. Since the early detection of endometrial carcinoma allows high opportunity for cure $^{17}$, the objective of this study was to evaluate the role of DWI with ADC value measurement in differentiating benign from malignant endometrial lesions with reference to histopathological diagnosis as a gold standard, we found that ADC value is an excellent non-invasive discriminatory test that produce high sensitivity and specificity in detecting endometrial malignancy, this is especially helpful in those patients in whom D\&C or transvaginal biopsy is difficult or risky 
and also helpful in avoiding unnecessary surgical intervention for benign causes as well as avoiding delay in intervention for malignancy, i.e. it's helpful in stratifying patients who need subsequent invasive test and its urgency ${ }^{18}$.

Our study included 18 (38.3\%) malignant cases, the main source for referral of cases was the gynecologic oncology unit of Baghdad teaching hospital, which is considered a tertiary center dealing mainly with suspicious cases, this may account for the higher proportion of malignant cases out of total endometrial lesions that had been evaluated in our sample. All lesions had been examined with both routine pelvic MRI protocol and DWI, all lesions in malignant group show hyperintense signal on DWI so hyperintensity on high $b$ value DWI is useful to delineate endometrial cancer however it cannot differentiate benign from malignant lesions as some benign lesions $(51.7 \%$ of benign lesions in this study) also show hyperintense signal and the rest show iso/hypointense signal. Fortunately, ADC map reconstructed from DWI provide quantitative evaluation and we found a statistically significant difference between ADC value of malignant group (mean ADC value $0.713 \times 10-3 \mathrm{~mm} 2 / \mathrm{sec}$ ) and that of benign group (mean $\mathrm{ADC}$ value $1.518 \times 10-3$ $\mathrm{mm} 2 / \mathrm{sec}$ ) with $\mathrm{P}$ value $<0.01$, this was in accordance with previously published studies. Shen et al.(18) in their study on 31 patients, from whom 24 cases were proved to have endometrial cancer, had found a significant difference between the malignant and benign groups with mean $\mathrm{ADC}$ value for each group was 0.864 and $1.277 \times 10-3 \mathrm{~mm} 2 / \mathrm{sec}$ respectively $(\mathrm{P}$ value 0.0058), the same statistical significance was found by Tamai et $\mathrm{al}^{19}$. in a study conducted in Japan on 18 cases of endometrial cancer and 12 with normal endometrium were the mean $\mathrm{ADC}$ value (x $10-3 \mathrm{~mm} 2 / \mathrm{sec}$ ) was $0.88 \pm 0.16$ for malignant group and $1.53 \pm 0.10$ for benign group, and by Takeuchi et $\mathrm{al}^{20}$ who over three years evaluated 67 endometrial lesion including 22 benign lesions and 45 cancers, the ADC value for benign and cancer cases was $1.58 \pm 0.36$ and $0.84 \pm 0.19 \quad$ x $\quad 10-3 \quad \mathrm{~mm} 2 / \mathrm{sec}$ respectively. However, our mean ADC value for malignant lesions was slightly lower than in these studies and this may be attributed to the method of choosing ROI, we try to compare all imaging sequences with $\mathrm{ADC}$ map and select the area corresponding to the region of highest SI on DWI plus avoiding cystic, necrotic and hemorrhagic area rather than selecting maximum area in the lesion.

Regarding the subdivisions of benign group, we found no significant difference in ADC value between polyps, hyperplasia and other benign entities ( $P$ value 0.342), similar finding had been shown by Bakir et $\mathrm{al}^{21}$ in Turkey in their retrospective study on 79 benign lesions out of 140 patients evaluated over 6 years, this may be explained by the fact that benign lesions share the same features of having less cellularity and higher extracellular water content than malignant lesions ${ }^{22}$.

Concerning ADC cut off value between benign and malignant group, we can choose between two cut off values either $0.874 \times 10-3 \mathrm{~mm} 2 / \mathrm{sec}$ which gives $100 \%$ specificity but the sensitivity is $94 \%$ that means we will have $6 \%$ false negative rate (malignant cases falsely labelled as benign), or $0.976 \times 10-3 \mathrm{~mm} 2 / \mathrm{sec}$ which gives $100 \%$ sensitivity but the specificity is $89.6 \%$ that means we will have $10.4 \%$ of benign cases falsely labelled as positive. Since we deal with a cancer, we believe it's better to have $100 \%$ sensitivity in order not to miss a malignant case and we can accept lower specificity and expose few benign cases to further evaluation, so the cut off value $0.976 \times 10-3 \mathrm{~mm} 2 / \mathrm{sec}$ seems to be more suitable. This cut off value was comparable to that reported by Karakas et $\mathrm{al}^{23}$ (ADC cut off value $<0.923 \times 10-3$ $\mathrm{mm} 2 / \mathrm{sec}$ ) however its sensitivity and 
specificity were lower $(90 \%$ and $81.8 \%$ respectively), other studies reported a little bit higher cut off values, Takeuchi et $\mathrm{al}^{20}$ reported a $96 \%$ sensitivity, 95\% specificity, 98\% PPV, and 91\% NPV when used $1.2 \times 10-3 \mathrm{~mm} 2 / \mathrm{sec}$ as a cut off value, while Kececi et $\mathrm{al}^{24}$ state a cut off value of $1.10 \times 10-3 \mathrm{~mm} 2 / \mathrm{sec}$ that results in $85.7 \%$ sensitivity and $92.8 \%$ specificity. This variation may be attributed to difference in range of ADC values in each study which in turn may be affected by sample size, types of benign lesions included, the grade of carcinoma (low, intermediate or high grade), strength of diffusion gradients and different MRI system manufacturers.

In our study, according to the selected cut off value, we had 3 benign cases with ADC value slightly below the cut off $(0.91,0.95$ and $0.9 \times 10-3 \mathrm{~mm} 2 / \mathrm{sec})$ i.e. false positive cases, the histopathological result in two of them, after hysterectomy, was endometrial polyp and in the 3rd, after D\&C, was endometrial hyperplasia with atypia. From histological point of view, polyps composed from mixture of three components that are: endometrial glands, stroma of dense fibrous tissue and thick walled blood vessels ${ }^{25,26}$, the difference in proportion of each element may explain the difference in ADC value especially one of our two cases shows abundant fibrotic stroma on histological examination with low T2 SI and isohypointense signal on visual assessment of DWI so it is from the start not fitting the definition of restricted diffusion regardless of $\mathrm{ADC}$ value. Regarding the 3 rd case whether or not the presence of atypia with hyperplasia can be the cause of low ADC value, cannot be inferred from this study and we believe it needs a dedicated study targeted specifically on this subject to include many cases of diverse types of hyperplasia.

Combining DWI with conventional routine pelvic MRI protocol seems promising. If we kept in mind that the definite diagnostic criteria for endometrial carcinoma on conventional MRI protocol is the demonstration of myometrial invasion ${ }^{4,17}$, that means we may misinterpret early endometrial carcinoma as benign lesion or overlook foci of malignant transformation on background of hyperplasia if we depend solely on morphological criteria, since DWI is a functional imaging it may detect early disease before myometrial invasion $^{27}$.

The limitation to our study was the presence of lesions diagnosed only by $\mathrm{D} \& \mathrm{C}$, which is unavoidable in benign lesions given the fact that hysterectomy is not mandatory for these lesions.

In conclusions DWI with ADC value measurement is a valuable non-invasive diagnostic test aiding to differentiate benign from malignant uterine endometrial cavity lesions, using a cut off ADC value of $0.976 \times 10-3 \mathrm{~mm} 2 / \mathrm{sec}$ yielding $100 \%$ sensitivity and $89.6 \%$ specificity for diagnosing endometrial carcinoma, however, ADC value measurement is not helpful to determine the subtype of benign endometrial lesions (polyp, hyperplasia and others) since it shows no significant difference among them.

Acknowledgment: Special thanks to Dr. Loay Almusawi for his great assistance in the statistical analysis of the results, and to technicians in the MR unit of Oncology Teaching Hospital, they facilitated the examination of patients and provided great collaboration \& technical help. 
Sample case from the study

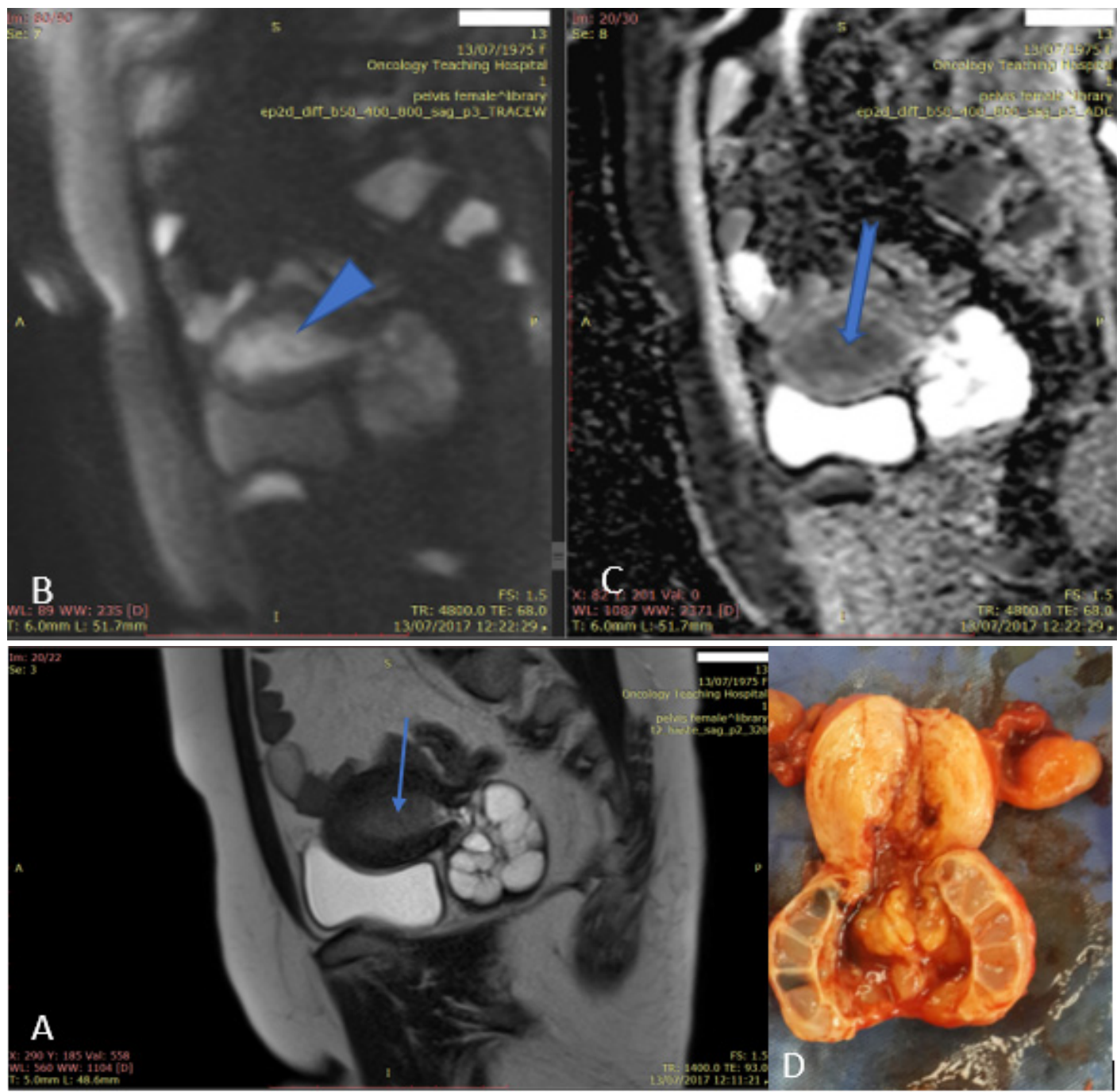

A 42 year old unmarried female present with abnormal uterine bleeding for 2 years, A. On T2 the endometrium signal was hypo-isointense (arrow), B. DWI: high SI (arrowhead), C. dark signal on ADC with low value $0.65 \times 10-3 \mathrm{~mm} 2 / \mathrm{sec}$ (arrow). on histopathological analysis the result was atypical complex hyperplasia with low grade endometrial adenocarcinoma of endometrioid type with focal squamous differentiation invading the myometrium more than inner half, the cervix shows chronic cystic cervicitis. D. Gross specimen.

\section{References}

1- Nalaboff KM, Pellerito JS, Ben-Levi E. Imaging the Endometrium: Disease and Normal Variants. RadioGraphics. 2001;21(6):1409-1424.

2- Kierans AS, Bennett GL, Haghighi M, Rosenkrantz AB. Utility of conventional and diffusionweighted MRI features in distinguishing benign from malignant endometrial lesions. European Journal of Radiology. 2014;83(4):726-732.

3- Curtis MG, Linares ST, Antoniewicz L, Glass RH. Glass office gynecology. Philadelphia: Lippincott Williams \& Wilkins; 2014. P. 583.

4- Shaaban AM, Menias CO, Tubay MS, Rezvani M, Farouk ESR, Woodward PJ. Diagnostic imaging: gynecology. Philadelphia, PA: Elsevier; 2015.

5- Sala E, Wakely S, Senior E, Lomas D. MRI of Malignant Neoplasms of the Uterine Corpus and Cervix. American Journal of Roentgenology. 2007;188(6):1577-87. 
6- Whittaker CS, Coady A, Culver L, Rustin G, Padwick M, Padhani AR. Diffusion-weighted MR Imaging of Female Pelvic Tumors: A Pictorial Review. RadioGraphics. 2009;29(3):759-74.

7- Qayyum A. Diffusion-weighted Imaging in the Abdomen and Pelvis: Concepts and Applications. RadioGraphics. 2009;29(6):1797-1810.

8- Patterson DM, Padhani AR, Collins DJ. Technology Insight: water diffusion MRI - a potential new biomarker of response to cancer therapy. Nature Clinical Practice Oncology. 2008;5(4):220-233.

9- Grant LA, Griffin N. Grainger \& Allison's Diagnostic Radiology Essentials. London: Churchill Livingstone/ Elsevier; 2013. P. 348-349.

10- Thoeny HC, Keyzer FD. Extracranial applications of diffusion-weighted magnetic resonance imaging. European Radiology. 2007Jun;17(6):1385-1393.

11- Koh D-M, Collins DJ. Diffusion-Weighted MRI in the Body: Applications and Challenges in Oncology. American Journal of Roentgenology. 2007;188(6):1622-1635.

12- Bonekamp S, Corona-Villalobos CP, Kamel IR. Oncologic applications of diffusion-weighted MRI in the body. Journal of Magnetic Resonance Imaging. 2012;35(2):257-279.

13- Malayeri AA, Khouli RH, Zaheer A, et al. Principles and Applications of Diffusion-weighted Imaging in Cancer Detection, Staging, and Treatment Follow-up. RadioGraphics. 2011;31(6):1773-1791. 14- Moore WA, Khatri G, Madhuranthakam AJ, Sims RD, Pedrosa I. Added Value of DiffusionWeighted Acquisitions in MRI of the Abdomen and Pelvis. American Journal of Roentgenology. 2014;202(5):995-1006.

15- Dale BM, Braithwaite AC, Boll DT, Merkle EM. Field Strength and Diffusion Encoding Technique Affect the Apparent Diffusion Coefficient Measurements in Diffusion-Weighted Imaging of the Abdomen. Investigative Radiology. 2010;45(2):104-108.

16-Padhani AR, Liu G, Mu-Koh D, et al. Diffusion-Weighted Magnetic Resonance Imaging as a Cancer Biomarker: Consensus and Recommendations. Neoplasia. 2009Feb;11(2):102-125.

17- Khati N, Glanc P, Bhosale P, et al. ACR Appropriateness Criteria ${ }^{\circledR}$ Abnormal vaginal bleeding. Available at https://acsearch.acr.org/docs/69458/Narrative. American College of Radiology. Accessed Jan/ 22/ 2018.

18-Shen S-H, Chiou Y-Y, Wang J-H, et al. Diffusion-Weighted Single-Shot Echo-Planar Imaging with Parallel Technique in Assessment of Endometrial Cancer. American Journal of Roentgenology. 2008;190(2):481-488.

19- Tamai K, Koyama T, Saga T, et al. Diffusion-weighted MR imaging of uterine endometrial cancer. Journal of Magnetic Resonance Imaging. 2007;26(3):682-687.

20-Takeuchi M, Matsuzaki K, Nishitani H. Diffusion-weighted magnetic resonance imaging of endometrial cancer: differentiation from benign endometrial lesions and preoperative assessment of myometrial invasion. Acta Radiologica. 2009;50(8):947-953.

21-Bakir B, Sanli S, Bakir VL, et al. Role of diffusion weighted MRI in the differential diagnosis of endometrial cancer, polyp, hyperplasia, and physiological thickening. Clinical Imaging. 2017;41:86-94.

22- Levy A, Medjhoul A, Caramella C, et al. Interest of diffusion-weighted echo-planar MR imaging and apparent diffusion coefficient mapping in gynecological malignancies: A review. Journal of Magnetic Resonance Imaging. 2011;33(5):1020-1027.

23-Karakas O, Karakas E, Dogan F, et al. Diffusion-weighted MRI in the differential diagnosis of uterine endometrial cavity tumors. Wiener klinische Wochenschrift. 2015;127(7-8):266-273.

24- Kececi IS, Nural MS, Aslan k, Danaci M, kefeli M, Tosun M. Efficacy of diffusion-weighted magnetic resonance imaging in the diagnosis and staging of endometrial tumors. Diagnostic and interventional imaging. 2016;97:177-186.

25-Özen Ö, Kiliçarslan A, Arat Z, Demirhan B. Diagnostic utility of glandular arrangement in endometrial polyps. Turkish Journal of Pathology. 2006;22(1):21-25.

26- Hase S, Mitsumori A, Inai R, et al. Endometrial Polyps: MR Imaging Features. Acta Medica Okayama. 2012Dec;66(6):475-485.

27- Takeuchi M, Matsuzaki K, Harada M, Tokushima JP. Endometrial carcinoma: Diagnostic strategy by using advanced MR techniques [Internet]. Educational exhibit at: European Congress of Radiology; 2013 Mar [cited 20170ct24]. Available from:

http://posterng.netkey.at/esr/viewing/index.php?module=viewing_poster\&doi=10.1594/ecr2013/C-1349. 